\title{
Weak gravitational lensing with the Square Kilometre Array
}

M. L. Brown ${ }^{*}{ }^{1}$ D. J. Bacon, ${ }^{2}$ S. Camera, ${ }^{1}$ I. Harrison, ${ }^{1}$ B. Joachimi, ${ }^{3}$ R. B. Metcalf, ${ }^{4}$ A. Pourtsidou, ${ }^{2}$ K. Takahashi, ${ }^{5}$ J. A. Zuntz, ${ }^{1}$ F. B. Abdalla,,${ }^{3,6}$ S. Bridle, ${ }^{1}$ M. Jarvis, ${ }^{7}$ T. D. Kitching, ${ }^{3}$ L. Miller, ${ }^{7}$ P. Patel ${ }^{8}$

${ }^{1}$ Jodrell Bank Centre for Astrophysics, School of Physics and Astronomy, University of Manchester, Oxford Road, Manchester M13 9PL, UK

${ }^{2}$ Institute of Cosmology and Gravitation, University of Portsmouth, Burnaby Road, Portsmouth PO1 3FX, UK

${ }^{3}$ Department of Physics and Astronomy, University College London, Gower Street, London, WC1E 6BT, UK

${ }^{4}$ Dipartimento di Fisica e Astronomia, Universitá di Bologna, viale B. Pichat 6/2 , 40127, Bologna, Italy

${ }^{5}$ Faculty of Science, Kumamoto University, 2-39-1 Kurokami, Kumamoto 860-8555, Japan

${ }^{6}$ Department of Physics and Electronics, Rhodes University, PO Box 94, Grahamstown, 6140, South Africa

${ }^{7}$ Astrophysics, Department of Physics, University of Oxford, Oxford OX1 3RH, UK

${ }^{8}$ Department of Physics, University of Western Cape, Cape Town 7535, South Africa

Email: m.1.brown@manchester.ac.uk

\begin{abstract}
We investigate the capabilities of various stages of the SKA to perform world-leading weak gravitational lensing surveys. We outline a way forward to develop the tools needed for pursuing weak lensing in the radio band. We identify the key analysis challenges and the key pathfinder experiments that will allow us to address them in the run up to the SKA. We identify and summarize the unique and potentially very powerful aspects of radio weak lensing surveys, facilitated by the SKA, that can solve major challenges in the field of weak lensing. These include the use of polarization and rotational velocity information to control intrinsic alignments, and the new area of weak lensing using intensity mapping experiments. We show how the SKA lensing surveys will both complement and enhance corresponding efforts in the optical wavebands through cross-correlation techniques and by way of extending the reach of weak lensing to high redshift.
\end{abstract}

Advancing Astrophysics with the Square Kilometre Array

June 8-13, 2014

Giardini Naxos, Italy

\footnotetext{
* Speaker.
} 


\section{Background}

\subsection{Cosmology with weak lensing surveys}

Weak gravitational lensing is the coherent distortion in the shapes of distant galaxies due to the deflection of light rays by intervening mass distributions. Measurements of the effect on large scales is termed "cosmic shear" and has emerged as a powerful probe of late-time cosmology over the last 15 years (see e.g. Heymans et al. 2013 for recent results from the CFHTLenS survey). Since gravitational lensing is sensitive to the total (i.e. dark plus baryonic) matter content of the Universe, it has great potential as a very robust cosmological probe, to a large degree insensitive to the complications of galaxy formation and galaxy bias. One of the most promising aspects of weak lensing measurements is their combination with redshift information: such measurements are then a sensitive probe of both the geometry of the Universe and of the evolution of structure over the course of cosmic time. In turn, these latter effects are dependent on the nature of the dominant dark energy component in the Universe and/or on modifications to the theory of General Relativity on large scales.

The observed distortions in the shapes of distant galaxies yields an estimate of the lensing shear field, $\gamma$. Since gravity is a potential theory, the shear at angular position $\theta$ can be related to a lensing potential $(\psi)$ as

$$
\gamma_{i j}(\theta)=\left(\delta_{i} \delta_{j}-\frac{1}{2} \delta_{i j}^{K} \delta^{2}\right) \psi(\theta),
$$

where $\delta_{i} \equiv r\left(\delta_{i j}-\hat{r}_{i} \hat{r}_{j} \nabla_{i}\right)$ is a dimensionless, transverse differential operator, and $\delta^{2}=\delta_{i} \delta^{j}$ is the transverse Laplacian. The indices $(i, j)$ each take the values $(1,2)$. In equation (1.1) we have assumed a flat sky which is an excellent approximation for the scales of interest (i.e. from $\sim 100$ $h^{-1} \mathrm{kpc}$ to $\sim 100 h^{-1} \mathrm{Mpc}$ ). The lensing potential can in turn be related to the 3-d gravitational potential, $\Phi(\mathbf{r})$ by (e.g. Kaiser 1998; Hu 2000)

$$
\psi(\theta)=\frac{2}{c^{2}} \int_{0}^{r} d r^{\prime}\left(\frac{r-r^{\prime}}{r r^{\prime}}\right) \Phi\left(\mathbf{r}^{\prime}\right)
$$

where $r$ is the comoving distance to the sources.

In the limit of weak lensing $(\gamma<<1)$, and in the absence of intrinsic correlations in galaxy ellipticities, one can form an unbiased estimator for the shear field at a given sky position from the average of the observed galaxy ellipticities at that position. Since the shear field induced by large scale structure is small (typically $\sim$ a few $\%$ ) compared to the intrinsic dispersion in galaxy ellipticities $\left(\sigma_{\varepsilon} \sim 0.3\right)$ one needs to average over many background galaxies in order to obtain a precise measurement. Measurements of cosmic shear can be affected by a number of instrumental and astrophysical systematic effects. The primary insturmental effect of concern is anisotropies in the point spread function (or beam) of the telescope which can mimic a cosmic shear signal. On the astrophysical side, alignments in the intrinsic shapes of galaxies can also mimic a cosmic shear signal. Consequently a great deal of effort is currently focused on careful instrument design for the next generation of weak lensing surveys as well as theoretical modelling and numerical simulations of intrinsic alignment effects.

Observationally, to date the field of weak lensing has largely been the preserve of optical surveys due to the much larger number densities of background galaxies achieved in such surveys. 
However, this will change with the advent of the Square Kilometre Array which will reach number densities of well-detected and well-resolved galaxies of up to $\sim 5$ galaxies $\operatorname{arcmin}^{-2}$ over several thousand $\mathrm{deg}^{2}$ in Phase 1 , and $\sim 10$ galaxies $\operatorname{arcmin}^{-2}$ over $3 \pi$ steradians in Phase 2 . In addition, as described in this chapter, the radio offers truly unique approaches to measuring weak lensing that are (i) not available to optical surveys and (ii) potentially extremely powerful in minimizing the most worrying systematic effects in weak lensing cosmology.

SKA surveys will also extend the reach of weak lensing beyond that of the optical megasurveys of LSST and Euclid. First the typical redshifts probed by SKA surveys will go beyond that of optical surveys. Secondly the SKA offers the prospects of measuring the weak lensing distortion in $21 \mathrm{~cm} \mathrm{HI}$ intensity maps. Such an intensity mapping lensing survey will not only extend to the high-redshifts inaccessible to optical surveys but will also include precise redshift information to accompany the distortion signal. This signal will fill the gap between traditional galaxy lensing signal (where the sources are typically located at $z \sim 1$ ) and the CMB lensing signal originating at $z=3000$. It thus holds the promise of yielding very precise cosmological constraints on the evolution of structure during early times where structures are better described with linear physics and can provide unique insight into so-called "early dark energy" models which exhibit observable signatures at early times.

\subsection{Radio weak lensing studies to date}

To date, the application of weak lensing analyses to radio data has been rather limited due to the relatively low number density of background galaxies achieved in large scale radio surveys. The only major weak lensing analysis of a radio survey was performed in Chang et al. (2004) who detected a cosmic shear signal in the Faint Images of the Radio Sky at Twenty cms (FIRST) survey (Becker et al. 1995), conducted with the Very Large Array (VLA). Although the number density of sources in FIRST was low by optical standards (FIRST contains $\sim 90$ sources $\mathrm{deg}^{-2}$ compared with typically $\sim 10$ sources $\operatorname{arcmin}^{-2}$ in deep optical lensing surveys), a detection of cosmic shear on large scales was achieved by virtue of the large survey area covered $\left(\sim 10,000 \mathrm{deg}^{2}\right)$.

The analysis of Chang et al. (2004) made use of the shapelets method (Chang \& Refregier 2002; Refregier \& Bacon 2003) to measure the shapes of the FIRST sources directly from the $u v$ interferometric data and included a thorough treatment of the possible systematics that can affect radio-based lensing shear estimates in particular.

More recently, Patel et al. (2010) revisited the idea of radio-based lensing shear estimation through a re-analysis of the combined VLA + MERLIN observations of the Hubble Deep Field North (Muxlow et al. 2005). This work presented an implementation of image-based shapelets for shear estimation and also highlighted the advantages of cross-correlating radio-based and opticalbased shear estimates obtained over the same area of sky.

\subsection{The path to SKA weak lensing}

A great deal of algorithm development and new analysis techniques are required in order to develop the field of radio weak lensing beyond the initial studies mentioned above. Key areas where work is required are the development of shape and/or shear estimation techniques that are well suited to radio interferometer datasets, the development of cross-correlation techniques for 
combining optical and radio-based shear estimates and the development and demonstration of novel radio-specific lensing approaches making use of polarization and rotational velocity information. A number of SKA pathfinder and precursor telescopes lend themselves naturally to developing several of these areas.

A key pathfinder telescope for demonstrating the long-baseline high resolution observations suited for weak lensing is the e-MERLIN interferometer based in the UK. A number of e-MERLIN legacy programs are well-suited to pathfinding the techniques for SKA weak lensing. Of particular note are the e-MERGE ${ }^{1}$ and SuperCLASS ${ }^{2}$ projects. e-MERGE is a multi-tiered project that (amongst other things) will image the GOODS-N field to $0.5 \mu \mathrm{Jy} \mathrm{rms} \mathrm{in} \mathrm{the} \mathrm{central} 100 \mathrm{arcmin}^{2}$ and to $1 \mu \mathrm{Jy} \mathrm{rms}$ in the surrounding $800 \mathrm{arcmin}^{2}$. The SuperCLASS project complements e-MERGE. Its primary science driver is to detect the weak lensing effects of a supercluster of galaxies located at $z=0.2$. SuperCLASS will image a $1.75 \mathrm{deg}^{2}$ field to $4 \mu \mathrm{Jy} \mathrm{rms}$. Both e-MERGE and SuperCLASS will perform their primary observations at $1.4 \mathrm{GHz}$ with a resolution of 200 mas. Both will therefore act as training experiments for demonstrating shape measurement and shear extraction algorithms on high-resolution radio data, on the path to the SKA.

A further opportunity exists to refine and test radio lensing analyses with the upgraded JVLA. A series of sky surveys $\left(\right.$ VLASS $^{3}$ ) are currently being discussed for the JVLA and these will potentially include a deep fields component. If conducted with an array configuration that includes long baselines, these could be extremely powerful for pathfinding weak lensing techniques in the radio over larger survey areas, approaching $10 \mathrm{deg}^{2}$ (Brown et al. 2013).

Other opportunities for demonstrating weak lensing techniques in the run up to the SKA include LOFAR surveys ${ }^{4}$ (when the international baselines are included) and the CHILES $^{5}$ and CHILES con $\mathrm{Pol}^{6}$ surveys on the JVLA. The LOFAR surveys will be critical for testing weak lensing techniques at low frequencies while the CHILES and CHILES con Pol surveys will prove useful for testing novel ideas for radio lensing including the use of polarization information and HI rotational velocity measurements (see Section 2.4).

\section{Cosmic shear with radio continuum surveys}

\subsection{Accessing the largest scales and the highest redshifts}

One of the potentially most exciting aspects of weak lensing surveys with the SKA will be the extra reach in terms of accessing the largest scales in the Universe and going beyond the reach of other weak lensing surveys in terms of the redshifts that are probed. We illustrate this in Figs. 1-3 where the redshift distributions of weak lensing source galaxies and the corresponding forecasted errors on a set of tomographic shear power spectra are presented. We present these forecasts for a 2-year continuum survey with the SKA at three different stages of development - an early phase of SKA1 comprising 50\% of the envisaged sensitivity targeting a survey area of $1000 \mathrm{deg}^{2}$; the

\footnotetext{
${ }^{1}$ http://www.e-merlin.ac.uk/legacy/projects/emerge.html

${ }^{2}$ http://www.e-merlin.ac.uk/legacy/projects/superclass.html

${ }^{3} \mathrm{https}: / /$ science.nrao.edu/science/surveys/vlass

${ }^{4}$ http://lofar.strw.leidenuniv.nl

${ }^{5}$ http://www.mpia-hd.mpg.de/homes/kreckel/CHILES/index.html

${ }^{6} \mathrm{http}: / /$ www.aoc.nrao.edu/ chales/chilesconpol/
} 
Table 1: Observational parameters used to produce the power spectrum and cosmological parameter forecasts in Figs. 1-4.

\begin{tabular}{lccc}
\hline Survey & $A_{\text {sky }}\left(\mathrm{deg}^{2}\right)$ & $n_{\text {gal }}\left(\operatorname{arcmin}^{-2}\right)$ & $z_{m}$ \\
\hline \hline SKA1-early & 1000 & 3.0 & 1.0 \\
VST-KiDS & 1500 & 7.5 & 0.6 \\
\hline SKA1 & 5000 & 2.7 & 1.0 \\
DES & 5000 & 6.0 & 0.6 \\
\hline SKA2 & 30940 & 37 & 1.6 \\
Euclid & 15000 & 30 & 0.9 \\
\hline
\end{tabular}

full SKA1 surveying $5000 \mathrm{deg}^{2}$, and a SKA2 survey covering $3 \pi$ steradians. To generate these forecasts, we have adopted the performance specifications for SKA1 outlined in Braun (2013). In particular, we have modeled the performance of Band 2 of the SKA-Mid facility as under the current baseline design, this telescope and frequency band combination provides the most powerful survey speed for the high angular resolution observations required for weak lensing. We have further assumed an object detection threshold of $S / N>10$ and an angular resolution requirement of $\theta_{\text {res }}=0.5 \mathrm{arcsec}$. To model the redshift and flux dependence of the source population, we have made use of the SKA Design Studies (SKADS) simulations of Wilman et al. (2008), updated to match the galaxy number counts observed in the deepest radio surveys performed to date (Muxlow et al. 2005; Morrison et al. 2010; Schinnerer et al 2010). We have also assumed an RMS dispersion in intrinsic galaxy ellipticities of $\gamma_{\mathrm{rms}}=0.3$

For comparison on these plots, we also show the corresponding forecasts for optical weak lensing surveys that will be conducted over similar survey areas and on comparable timescales. Specifically, we consider the VST-KiDS (de Jong et al. 2013), the Dark Energy Survey ${ }^{7}$ and the Euclid satellite mission (Laureijs et al. 2011). The observational parameters adopted to produce these forecasts are summarized in Table 1. In all cases, the radio surveys extend to higher redshift than the corresponding optical probes. They thus hold the potential to probe the power spectrum at higher redshift providing a more sensitive lever arm with which to constrain the growth of structure over cosmic time.

Fig. 4 presents forecasted constraints on the matter density $\left(\Omega_{m}\right)$ and matter power spectrum normalization $\left(\sigma_{8}\right)$ cosmological parameters for the same envisaged SKA surveys as were adopted to generate Figs. 1-3. Note that these forecasts are presented for the case of the standard 6parameter $\Lambda \mathrm{CDM}$ model and no prior information is assumed - that is the projected constraints are coming solely from the envisaged SKA weak lensing survey.

To generate the constraints, we have computed a simple shear power spectrum covariance matrix from Takada \& Jain (2004), and we use the CosMOSIS cosmological parameter estimation code (Zuntz et al. 2014) to compute power spectra, parameter constraints, and marginalised contours. Note that no systematic errors are included in the analysis; errors are purely statistical. However, we have attempted to take into account anticipated knowledge and uncertainties regard-

\footnotetext{
${ }^{7} \mathrm{http}: / /$ www.darkenergysurvey.org
} 

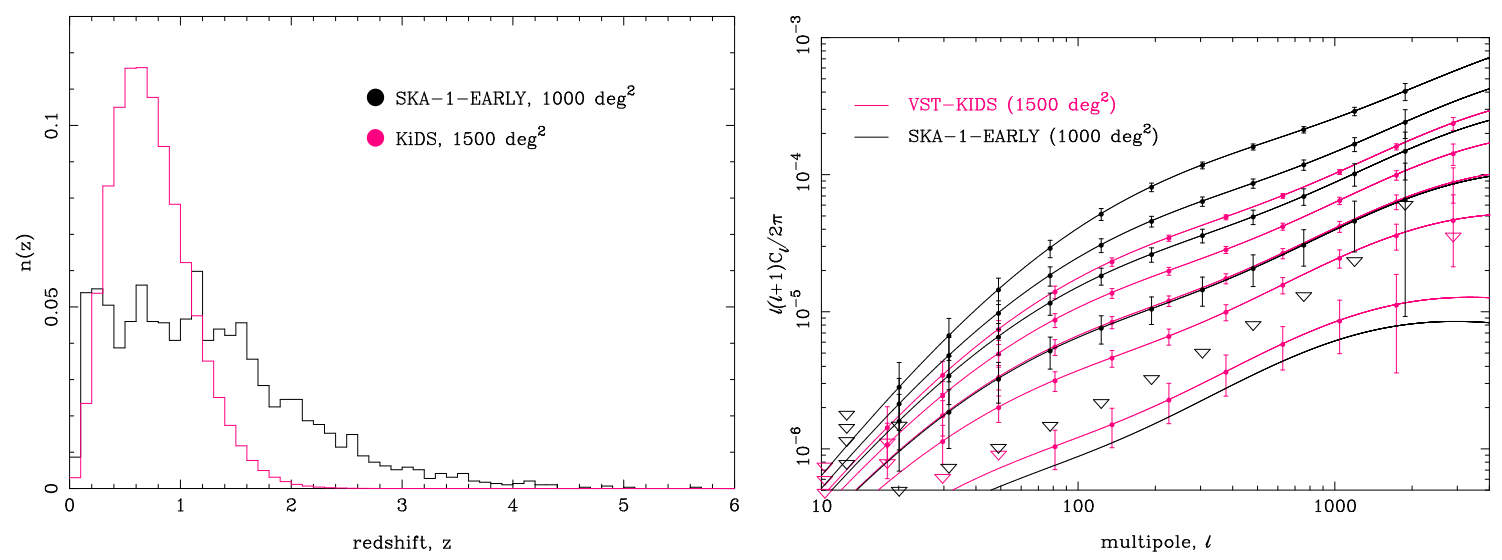

Figure 1: Left panel: The redshift distribution of source galaxies for a $1000 \mathrm{deg}^{2}$ weak lensing survey requiring 2 years observing time on the SKA1-early facility. Also shown is the redshift distribution for the $1500 \mathrm{deg}^{2}$ VST-KiDS optical lensing survey. The $n(z)$ extends to higher redshifts in the radio survey and probes a greater range of cosmic history. Right panel: The corresponding constraints on a 5-bin tomographic power spectrum analysis. For both experiments, we assumed an RMS dispersion in ellipticity measurements of $\gamma_{\mathrm{rms}}=0.3$ and the tomographic bins have been chosen such that the bins are populated with equal numbers of galaxies. Note how the radio survey extends to higher redshifts where the lensing signal is stronger and therefore easier to measure. Open triangles denote $1 \sigma$ upper limits on a bandpower. Note that only the auto power spectra in each bin are displayed though much cosmological information will also be encoded in the cross-correlation spectra between the different $z$-bins.
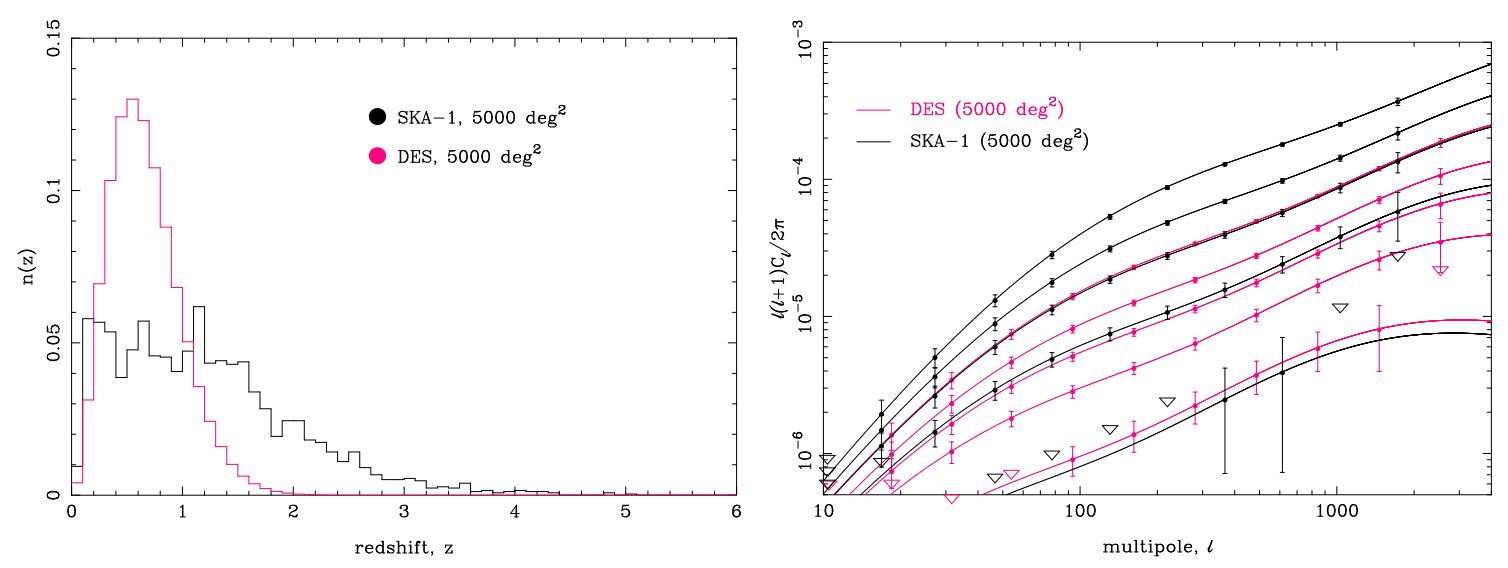

Figure 2: As Fig. 1 but for a $5000 \mathrm{deg}^{2}$ weak lensing survey requiring 2 years observing time on the full SKA1 facility. Also shown for comparison are the $n(z)$ distribution and forecasted power spectrum constraints for the $5000 \mathrm{deg}^{2}$ Dark Energy Survey.

ing photometric and spectroscopic redshift estimates for the background galaxy population. For SKA1-early, we have assumed that we have no spectroscopic redshift information and that we have photo- $z$ estimates from overlapping optical surveys with errors $\sigma_{z}=0.05(1+z)$ up to a limiting redshift of 1.5. To model the much larger uncertainties expected for the high- $z$ radio galaxies, we adopt $\sigma_{z}=0.3(1+z)$ so that a $z=2$ galaxy has a redshift uncertainty of $\pm \sim 1$. For SKA1, we additionally assume that we will have spectroscopic redshifts from overlapping $\mathrm{HI}$ observations for $15 \%$ of the $z<0.6$ population. Finally for SKA2, we assume we have spectroscopic redshifts 

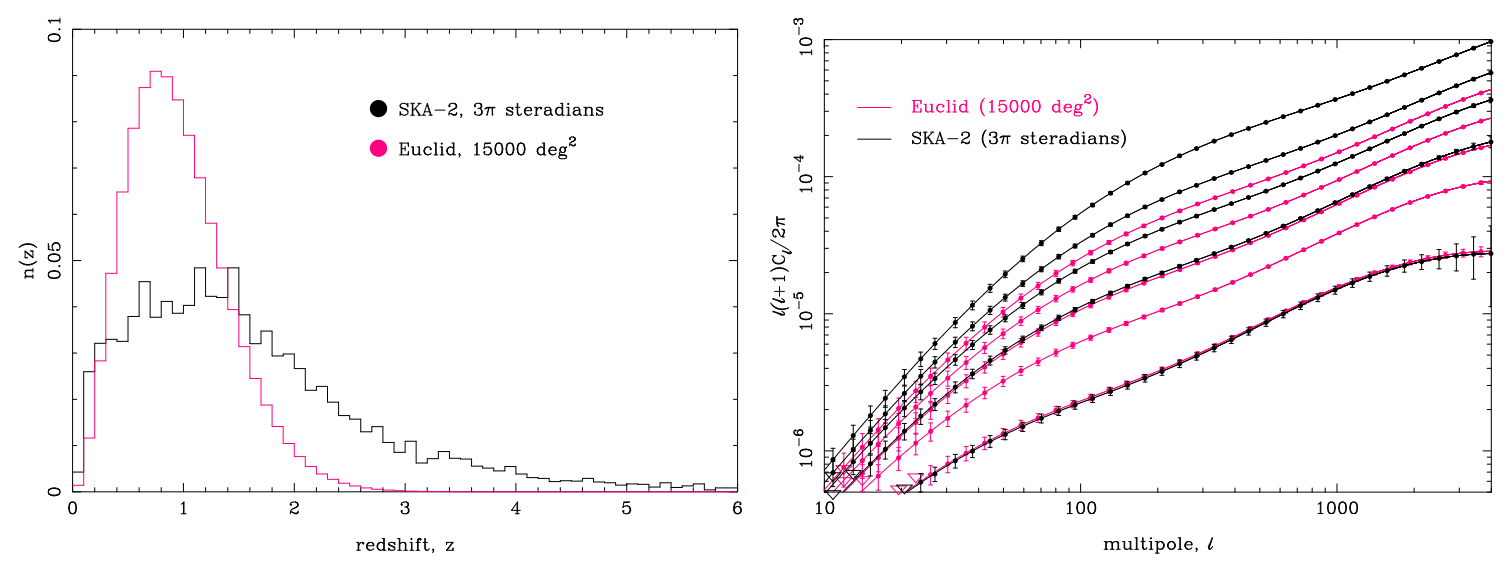

Figure 3: As Fig. 1 but for a $3 \pi$ steradian weak lensing survey requiring 2 years observing time on SKA2. Also shown for comparison are the $n(z)$ distribution and forecasted power spectrum constraints for the 15000 $\mathrm{deg}^{2}$ Euclid satellite mission.

for $50 \%$ of the $z<2.0$ population. The forecasts presented in Fig. 4 account for these redshift uncertainties.

We see from Fig. 4 that even the SKA1-early survey targeting the smallest sky area can provide competitive constraints on cosmological parameters - the forecasted constraints for the $1000 \mathrm{deg}^{2}$ SKA1-early survey are a factor of $\sim 5$ better than the tomographic weak lensing analysis of the current state-of-the-art CFHTLenS data (Heymans et al. 2013). We also see large improvements in the constraints obtainable with each subsequent stage of the SKA - the constraints obtainable with SKA1 are broadly comparable with the KiDS and DES optical surveys while SKA2 is competitive with Euclid.

Fig. 4 also demonstrates that our nominal choice of target survey areas for the three stages of the SKA are, broadly speaking, optimal choices from the point of view of constraining these cosmological parameters - for SKA1-early the $1000 \mathrm{deg}^{2}$ survey provides the strongest constraints, for SKA1 the $5000 \mathrm{deg}^{2}$ survey performs the best while for SKA2, the $3 \pi$ steradian survey provides the best constraints.

\subsection{The promise of radio observations to suppress weak lensing systematics}

Optical and radio surveys, such as Euclid and/or LSST and the SKA, have a particularly useful synergy in reducing and quantifying the impact of systematic effects which may dominate each survey alone on some scales. By cross-correlating the shear estimators from one of these surveys with those of the other, several systematic errors are mitigated.

We can see this by writing the contributions to an optical $(o)$ or radio $(r)$ shear estimator:

$$
\begin{aligned}
& \gamma^{(o)}=\gamma_{\mathrm{grav}}+\gamma_{\text {int }}^{(o)}+\gamma_{\text {sys }}^{(o)} \\
& \gamma^{(r)}=\gamma_{\text {grav }}+\gamma_{\text {int }}^{(r)}+\gamma_{\text {sys }}^{(r)},
\end{aligned}
$$

where $\gamma_{\text {grav }}$ is the gravitational shear we are seeking, $\gamma_{\text {int }}$ is the intrinsic ellipticity of the object, and $\gamma_{\text {sys }}$ are systematic errors induced by the telescope. If we correlate optical shears with optical 

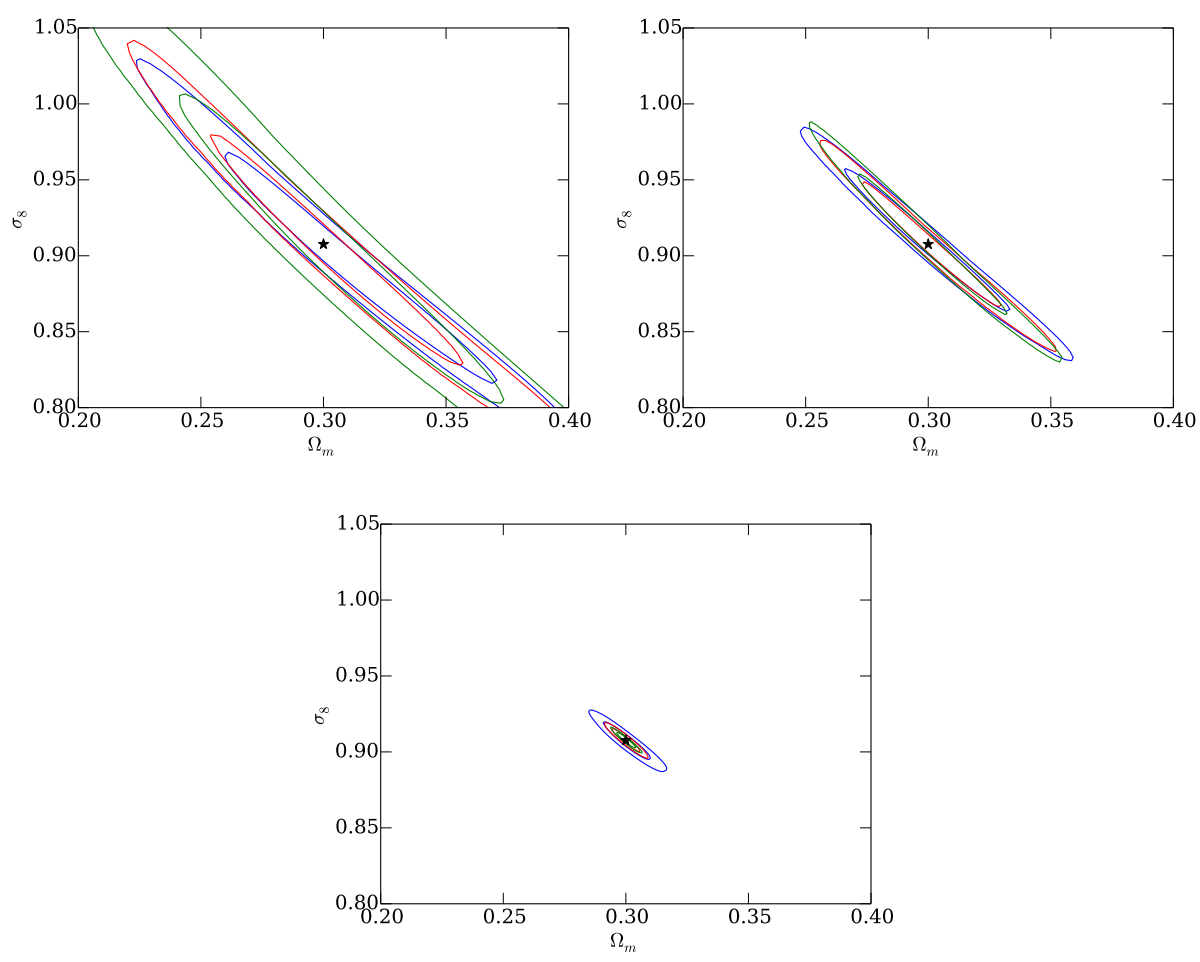

Figure 4: Forecasted constraints in the $\sigma_{8}-\Omega_{m}$ parameter space for 2-year continuum surveys using SKAMid/Band 2 with SKA1-early (upper left panel), SKA1 (upper right panel) and SKA2 (lower panel) performance parameters. For each of these cases, we present the constraints for survey areas of $1000 \mathrm{deg}^{2}$ (blue) $5000 \mathrm{deg}^{2}$ (red) and $3 \pi$ steradians (green).

shears, or radio shears with radio shears, we obtain terms like

$$
\langle\gamma \gamma\rangle=\left\langle\gamma_{\text {grav }} \gamma_{\text {grav }}\right\rangle+\left\langle\gamma_{\text {grav }} \gamma_{\text {int }}\right\rangle+\left\langle\gamma_{\text {int }} \gamma_{\text {int }}\right\rangle+\left\langle\gamma_{\text {sys }} \gamma_{\text {sys }}\right\rangle
$$

where the first term is the gravitational signal we seek, the second term is the GI intrinsic alignment (Hirata \& Seljak 2004), the third term is the II intrinsic alignment (e.g. Heavens et al. 2000), and the final term is the contribution from systematics. All of these terms could be similar size on certain scales, which is of damage to cosmological constraints. On the other hand, if we cross-correlate the optical shears with radio shears, we obtain

$$
\left\langle\gamma^{(o)} \gamma^{(r)}\right\rangle=\left\langle\gamma_{\text {grav }} \gamma_{\text {grav }}\right\rangle+\left\langle\gamma_{\text {grav }} \gamma_{\text {int }}^{(o)}\right\rangle+\left\langle\gamma_{\text {grav }} \gamma_{\text {int }}^{(r)}\right\rangle+\left\langle\gamma_{\text {int }}^{(o)} \gamma_{\text {int }}^{(r)}\right\rangle+\left\langle\gamma_{\text {sys }}^{(o)} \gamma_{\text {sys }}^{(r)}\right\rangle
$$

The second and third terms are the GI alignment (Hirata \& Seljak 2004), which still survives. However, the fourth term involves the correlation between optical and radio shapes, which will be less than that between one frequency alone as the emission mechanisms are different (c.f. Patel et al. 2010 where no correlation at zero lag was found). This term is therefore reduced. Most importantly, the fifth term involving systematics is expected to be zero, as the systematics in these two telescopes, which are of completely different design and function, are not expected to be correlated at all. We are therefore able to remove the dangerous systematics correlation from our shear analysis - and to gain an estimate of its magnitude in the autocorrelation case. 
Table 2: Requirements on multiplicative and additive biases on ellipticity measurement for proposed SKA weak lensing surveys to be dominated by statistical rather than systematics uncertainties. $Q$ is a global "quality factor" which we calculate from $m$ and $c$ following Voigt et al. (2010).

\begin{tabular}{lcccccc}
\hline Experiment & $A_{\text {sky }}$ & $n_{\text {gal }}$ & $z_{m}$ & $m<$ & $c<$ & $Q>$ \\
\hline \hline SKA1-early & 1000 & 3.0 & 1.0 & 0.014 & 0.0012 & 62 \\
SKA1-early & 5000 & 1.2 & 0.8 & 0.012 & 0.0011 & 79 \\
SKA1-early & 30940 & 0.35 & 0.5 & 0.011 & 0.0011 & 80 \\
\hline SKA1 & 1000 & 6.1 & 1.2 & 0.0090 & 0.00095 & 103 \\
SKA1 & 5000 & 2.7 & 1.0 & 0.0067 & 0.00082 & 140 \\
SKA1 & 30940 & 0.9 & 0.7 & 0.0058 & 0.00076 & 164 \\
\hline SKA2 & 1000 & 37 & 1.6 & 0.0031 & 0.00055 & 318 \\
SKA2 & 5000 & 23 & 1.4 & 0.0019 & 0.00043 & 523 \\
SKA2 & 30940 & 10 & 1.3 & 0.0012 & 0.00035 & 825 \\
\hline
\end{tabular}

A further systematic that is potentially problematic for weak lensing surveys are color-gradients (Voigt et al. 2012). However, galaxies typically have smooth spectral dependence at radio frequencies. This, combined with the ability to measure the spectral dependence of the beam very accurately, means that radio weak lensing surveys will be insensitive to this systematic.

\subsection{Shear measurement}

In order to extract the weak lensing science from the SKA, we will need to make highly accurate measurements of the shearing of background sources in real, noisy data. In optical experiments, this shear measurement process has typically consisted of measuring the ellipticity of individual galaxies and combining these measurements to form an estimate of the cosmic shear. A wide variety of galaxy shape measurement algorithms have been developed for this task and tested through the STEP and GREAT programmes (see e.g. Mandelbaum et al. 2014 and references therein) and experience with real data, honing the techniques and focusing research on areas which require improvement. As a consequence, shape measurement from optical data is a well-established field and techniques already developed are sufficiently advanced that shape measurement-induced systematics are likely to be sub-dominant to statistical errors in current and near future optical lensing surveys. These shape measurement systematics are often parameterised in terms of the additive bias $c$ and multiplicative bias $m$ on the ellipticity $e^{o b s}$ recovered from an input source with known true ellipticity $e^{\text {true: }}$

$$
e^{o b s}=(1+m) e^{t r u e}+c .
$$

A formalism is provided in Amara \& Refregier (2008) for calculating the $m$ and $c$ necessary for systematic errors to be sub-dominant, with requirements for a selection of SKA surveys shown in Table 2. However, these algorithms have almost solely been motivated by optical and NIR data, meaning their robustness to issues peculiar to radio data, in particular potential systematics from the non-linear deconvolution process necessary for imaging (and removal of sidelobe artifacts), is unclear. Of the two radio weak lensing studies so far performed, both measure shear by modeling data using the orthonormal shapelets basis set (Refregier \& Bacon 2003). Patel et al. (2010) use 
shapelets to model images reconstructed using the CLEAN algorithm, while Chang et al. (2004) take advantage of the fact that shapelets remain localised analytic functions under Fourier transformation to directly model the visibility plane and were able to make a $3.6 \sigma$ detection of cosmic shear. More recently, Patel et al. (2013) have applied image plane shapelets to simulations, allowing them to quantify the efficacy of the method, achieving values $m=0.176$ and $c=0.006$ for a simulated e-MERLIN observation. For SKA1, Patel et al. in this volume achieve $m=0.28$ and $c=0.001$ for image plane shapelets, comparable to requirements of $m<0.0054, c<0.0073$ for the SKA1 $5000 \mathrm{deg}^{2}$ survey, as well as providing an initial comparison of the relative performance of visibility plane shapelets.

A number of open problems still remain. Although initial steps have been taken, only a small part of the space of possible radio shear measurement techniques has so far been explored. Future work may be expected to take place with two main themes:

- Investigation of the ability of image reconstruction algorithms to produce images with the level of fidelity necessary for weak lensing cosmology and subsequent adaptation of known methods from optical/NIR weak lensing to radio data. High-fidelity image reconstruction is an active research topic in its own right, with many extensions and alternatives to traditional CLEAN and Maximum Entropy methods under development (e.g. Sutter et al. 2014; Carrillo et al. 2014 and references therein).

- Investigation of techniques which measure shear directly from interferometer visibilities. Such techniques have the advantage of avoiding systematics introduced by the non-linear deconvolution necessary for the imaging process and a visibility plane technique (shapelets in Chang et al. 2004) has been the only technique so far to successfully detect weak lensing in radio data. However, for the datasets produced by the SKA, computational challenges for visibility plane techniques will be great. Simultaneously fitting $\sim 5$ parameter models to large numbers of sources over large numbers of visibilities is likely to be unfeasible, though averaging and novel methods such as $u v$-stacking (Lindroos et al. 2014) have the potential to help. There also remains the open question as to whether resources to store the raw visibilities from SKA observations for later analysis will be available.

In the near-term, we expect to begin addressing these issues by extending previous Gravitational LEnsing Accuracy Testing (GREAT) challenges (e.g. Mandelbaum et al. 2014) to radio data with a radioGREAT challenge ${ }^{8}$. This will aim to better quantify the current status of shape measurement techniques for radio weak lensing, assess their behaviour across changing data parameters and spur new interest and developments in the field.

\subsection{Polarization and rotation velocities as indicators of intrinsic alignments}

One unique advantage of radio telescopes for weak lensing is the polarization information which can provide information on the intrinsic (unlensed) shapes of background galaxies. As described in Brown \& Battye (2011), the position angle of the integrated polarized emission from a background galaxy is unaffected by gravitational lensing. If the polarized emission (which is

\footnotetext{
${ }^{8}$ http://radiogreat.jb.man.ac.uk
} 
polarized synchrotron emission sourced by the galaxy's magnetic field) is also strongly correlated with the disk structure of the galaxy then measurements of the radio polarization position angle can be used as estimates of the galaxy's intrinsic (unlensed) position angle.

Such an approach could potentially have two key advantages over traditional weak lensing analyses. Firstly, the polarization technique can be used to effectively remove the primary astrophysical contaminant of weak lensing measurements - intrinsic galaxy alignments (Heavens et al. 2000; Catelan et al. 2001; Hirata \& Seljak 2004; Brown et al. 2002) - which are a severe worry for ongoing and future precision cosmology experiments based on weak lensing. Secondly, depending on the polarization properties of distant background disk galaxies, the polarization technique has the potential to reduce the effects of noise due to the intrinsic dispersion in galaxy shapes.

The power of the polarization technique in practice will depend on two key observational parameters: the scatter in the relationship between the observed polarization position angle and the intrinsic structural position angle of the galaxy $\left(\alpha_{\mathrm{rms}}\right)$ and the number of galaxies for which one can obtain accurate polarization measurements $\left(n_{\text {pol }}\right)$. These parameters depend on the details of the polarization properties of background galaxies (e.g. the mean polarization fraction, $\Pi_{\mathrm{pol}}$ ) which are currently not well known. There are some existing measurements for a sample of local spiral galaxies (Stil et al. 2009) which suggest $\alpha_{\text {rms }}<15^{\circ}$ and $\Pi_{\text {pol }}<20 \%$ although the sample is small. Note that it may be possible to select sub-samples of the total galaxy population to have particular polarization properties. For example, one could imagine that selecting only galaxies with high fractional polarization would yield a galaxy sub-sample with highly ordered magnetic fields which would consequently have a very tight correlation (low $\alpha_{\text {rms }}$ ) between the polarization orientations and the intrinsic structural position angles of the galaxies. Of course, such a sub-sample would also have a very low surface number density of galaxies. The polarization technique may therefore be better suited to probing the shear power spectrum on large scales where high number densities are not required.

A second novel idea that is well suited to radio observations is to use rotational velocity measurements to provide information about the intrinsic shapes of galaxies. The idea, first suggested by Blain (2002) and Morales (2006), is to measure the axis of rotation of a disk galaxy and to compare this to the orientation of the major axis of the galaxy disk image. In the absence of lensing, these two orientations should be perpendicular and measuring the departure from perpendicularity directly estimates the shear field at the galaxy's position. Such an analysis would require commensal HI line observations which could in principle be done at no extra cost in terms of telescope time. The rotation velocity technique shares many of the characteristics of the polarization approach described above - in the limit of perfectly well-behaved disk galaxies, it is also free of shape noise and it can also be used to remove the contaminating effect of intrinsic galaxy alignments. In practice, the degree to which the rotational velocity technique improves on standard methods will be dependent on observational parameters analogous to the ones for polarization discussed above. Recently, Huff et al. (2013) have proposed to extend this technique using the Tully-Fisher relation to calibrate the rotational velocity shear measurements and thus reduce the residual shape noise even further.

Both the polarization technique and the rotation velocity approach are currently being tested as part of the SuperCLASS, CHILES and CHILES con Pol projects. They offer great promise for reducing the impact of shape noise and intrinsic alignments in radio weak lensing surveys. 


\section{Weak lensing with HI Intensity Mapping}

Weak gravitational lensing of high redshift $21 \mathrm{~cm}$ emission has been the subject of several studies focusing on the Epoch of Reionization (EoR) observations. In Zahn \& Zaldarriaga (2006) and Metcalf \& White (2009) it was shown that if the EoR is at redshift $z \sim 8$ or later, a large telescope like the SKA could measure the lensing power spectrum and constrain the standard cosmological parameters. The authors extended the Fourier-space quadratic estimator technique, which was first developed in $\mathrm{Hu}$ (2001) for CMB lensing observations to three dimensional observables, i.e. the $21 \mathrm{~cm}$ intensity field $I(\theta, z)$. These studies did not consider $21 \mathrm{~cm}$ observations from redshifts after reionization when the average $\mathrm{HI}$ density in the Universe is much smaller.

$\mathrm{HI}$ intensity mapping is a technique that has been proposed for measuring the distribution of $\mathrm{HI}$ gas before and during reionization (see Pritchard et al. in this volume) and measuring the BAO at redshifts of order unity (Chang et al. 2008, 2010; Seo et al. 2010; Masui et al. 2010; Ansari et al. 2012; Battye et al. 2013; Chen 2012; Pober et al. 2013). In this technique, no attempt is made to detect individual objects. Instead the $21 \mathrm{~cm}$ emission is treated as a continuous three dimensional field. The angular resolution of the telescope need not be high enough to resolve individual galaxies which makes observations at high redshift possible with a reasonably sized telescope.

A recent study (Pourtsidou \& Metcalf 2014) extended the $21 \mathrm{~cm}$-intensity-mapping-lensing method further, taking into account the discreteness of galaxies, and investigated the possibility of measuring lensing at intermediate redshifts without resolving (in angular resolution) or even identifying individual sources. Here we present an improved analysis of the signal-to-noise expected from an SKA-like telescope array, and demonstrate the performance of the SKA-Mid facility for SKA1 and for SKA2.

One of the first objectives of a $21 \mathrm{~cm}$ lensing survey will be to measure the two-point statistics of the convergence field $\kappa\left(\vec{L}, z_{s}\right)$ or, equivalently, the displacement field $\delta \theta\left(\vec{L}, z_{s}\right)$, averaged over $z_{s}$. That is,

$$
C_{L}^{\delta \theta \delta \theta}=\frac{9 \Omega_{m}^{2} H_{0}^{3}}{L(L+1) c^{3}} \int_{0}^{z_{s}} d z P(k=L / r(z), z)[W(z)]^{2} / E(z),
$$

where $W(z)=\left(r\left(z_{s}\right)-r(z)\right) / r\left(z_{s}\right), E(z)=H(z) / H_{0}$. The expected error in the power spectrum $C_{L}^{\delta \theta \delta \theta}$ averaging over $\vec{L}$ directions in a band of width $\Delta L$ is given by

$$
\Delta C_{L}^{\delta \theta \delta \theta}=\sqrt{\frac{2}{(2 L+1) \Delta L f_{\text {sky }}}}\left(C_{L}^{\delta \theta \delta \theta}+N_{L}\right) .
$$

Here, $N_{L}$ is the derived $\delta \theta$ estimate reconstruction noise which involves the underlying dark matter power spectrum, the HI density $\Omega_{\mathrm{HI}}(z)$ as well as the HI mass moments up to 4th order and, of course, the thermal noise of the telescope (see Pourtsidou \& Metcalf 2014 for details). For the HI mass function we will first assume the most conservative scenario, i.e. a no evolution model using the results from the HIPASS survey (Zwaan et al. 2003), giving $\Omega_{\mathrm{HI}} \simeq 4.9 \times 10^{-4}$ (Model A). We will also consider a more realistic evolution scenario from Zhang \& Pen (2006) where $\Omega_{\mathrm{HI}}(z)=4.9 \times 10^{-4}(1+z)^{2.9} \exp (-z / 1.3)$. This is expressed through an evolution of the normalization of the Schechter function $\phi^{\star}$ (Model B).

Here we will concentrate on source redshift $z_{s} \sim 2$. The SKA1-Mid array operates in the frequency range $\sim 350-3050 \mathrm{MHz}$ divided in three bands (Dewdney et al. 2013). Our chosen 
$z_{s}$ corresponds to frequency $v_{s}=473 \mathrm{MHz}$ (Band 1). The power spectrum of the thermal noise of a SKA-like interferometer core array (using the uniform distribution approximation) is $C_{\ell}^{\mathrm{N}}=$ $\frac{(2 \pi)^{3} T_{\text {sys }}^{2}}{B t_{\text {obs }}^{2} f_{\text {cover }}^{2} \text { max }}(v)^{2}$, where the system temperature $T_{\text {sys }}=\left(40+1.1 T_{\text {sky }}\right) \mathrm{K}$ with $T_{\text {sky }}=60 \lambda^{-2.55}, B$ is the chosen frequency window, $t_{\mathrm{obs}}$ the total observation time, $D_{\text {tel }}$ the diameter (maximum baseline) of the array, $\ell_{\max }(\lambda)=2 \pi D_{\text {tel }} / \lambda$ is the highest multipole that can be measured by the array at frequency $v$ (wavelength $\lambda$ ), and $f_{\text {cover }}$ is the total collecting area of the telescopes $A_{\text {coll }}$ divided by $\pi\left(D_{\text {tel }} / 2\right)^{2}$. For SKA-Mid we can consider a $2 \mathrm{yr}$ observation time, $f_{\text {sky }} \sim 0.7$ (corresponding to $\sim 30,900 \mathrm{deg}^{2}$ survey area), and we further choose $B=20 \mathrm{MHz}$ and $\Delta L=36$. Keeping these values constant, in Fig. 5 we present forecasts for the displacement field power spectrum for source redshifts $z_{s}=2$ and $z_{s}=3$. For the Model A HI mass function, SKA1 does not have the sensitivity to measure the signal. However, for Model B we see a great improvement in the signal-to-noise ratio, such that the lensing signal can be well measured by SKA1 (Fig. 5, left panel). For SKA2, a precise characterization of the power spectrum is possible at multiple redshifts even in the conservation noevolution scenario (Model A, Fig. 5, right panel).
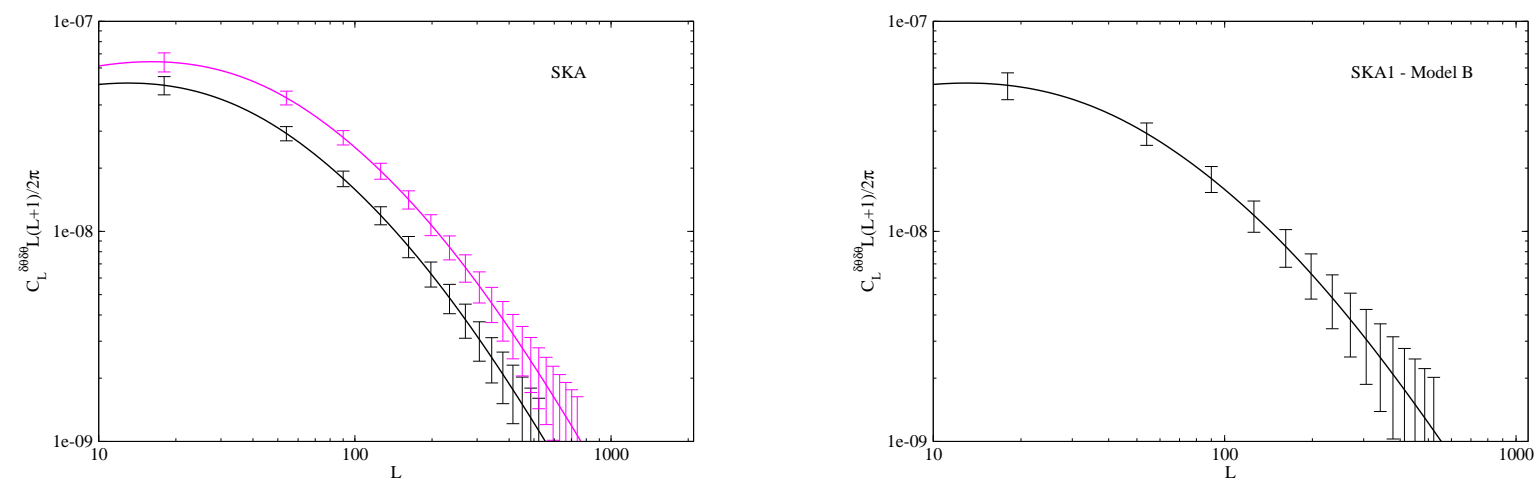

Figure 5: Left: Displacement field power spectrum for $z_{s}=2$ and the corresponding measurement errors using the SKA1-Mid specifications and Model B for the HI mass function. Right: Displacement field power spectrum for $z_{s}=2$ (solid black line) and $z_{s}=3$ (solid magenta line) and the corresponding measurement errors using the SKA2 specifications and Model A (no evolution) for the HI mass function. See the main text of Section 3 for details of the HI mass function adopted for Models A and B.

This technique should enable us to measure the lensing power spectrum at source redshifts well beyond those accessible with more traditional weak lensing surveys based on the shearing of individual galaxy images. This is a promising approach to investigating the possible evolution of dark energy or modified gravity at high redshift. In addition to measuring the power spectrum, the SKA-Low array should be able to map the dark matter distribution with high fidelity in a 5-by-5 degree field using this technique (see Pritchard et al. in this volume for details).

\section{Galaxy and cluster weak lensing}

In addition to probing large-scale structure, weak lensing can be used to measure the distribution of mass (both dark and bright) around individual galaxies, as well as other structures such as galaxy pairs, groups and clusters, or voids (Mandelbaum et al. 2006; Gillis et al. 2013; Melchior 
et al. 2014). Since the signal from an individual lens is too weak to be detectable, except for massive clusters, such "galaxy-galaxy" measurements are usually stacked to produce an average mass distribution around a certain type of lens objects. On large, linear scales galaxy-galaxy lensing constrains galaxy bias (e.g. Hoekstra et al. 2002). Moreover, interpreted as a galaxy position-shear cross-correlation it can provide additional cosmological constraints and valuable calibration of astrophysical systematics (Bernstein 2009; Joachimi \& Bridle 2010) and Kirk et al., this volume). On smaller scales galaxy-galaxy lensing is sensitive to the radial matter density profile of galaxy haloes as well as the abundance and distribution of substructure in its outskirts, all as a function of galaxy properties such as type, stellar mass, or velocity dispersion (van Uitert et al. 2013).

The SKA surveys will provide a unique angle to galaxy-galaxy weak lensing studies, with key contributions in the following areas:

- Competitive or superior statistical errors: These errors are driven by the number density of both lens and source galaxies (particularly on small scales) and the sample variance due to a finite sky coverage (particularly on large scales).

- Spectroscopic redshifts for new galaxy samples: To obtain unbiased measurements, a clean separation of lens and source samples is paramount. Spectroscopic redshifts for foreground objects allow for a clean and precise definition of lens samples and lens properties. Accurate redshifts for a subset of the source sample characterise the redshift distribution of background objects.

- Novel lens galaxy sample definitions: The information from radio wavelengths can be used to define new lens samples e.g. in terms of their radio luminosity, AGN activity, or gas abundance. This allows for the measurement of the relation between these properties and matter halo characteristics, e.g. the HI gas mass to halo mass ratio as a function of redshift.

To quantify this performance, we forecast expected errors on a one-parameter radial matter profile constrained via the average tangential shear measured in the range of $0.1 \mathrm{Mpc} / h$ to $1 \mathrm{Mpc} / h$ transverse separation from the lenses. We use a singular isothermal sphere (SIS) as a simple profile,

$$
\rho_{\mathrm{SIS}}(r)=\frac{\sigma_{v}^{2}}{2 \pi G r^{2}}
$$

where $r$ denotes three-dimensional distance, and where $\sigma_{v}$ is the velocity dispersion, which is the single free parameter. To facilitate comparison, we set $\sigma_{v}=250 \mathrm{~km} / \mathrm{s}$ for all calculations. The tangential shear signal for this profile is given by (assuming a spatially flat Universe, Bartelmann \& Schneider 2001)

$$
\left\langle\gamma_{+}\right\rangle\left(r_{p}\right)=2 \pi\left(\frac{\sigma_{v}}{c}\right)^{2} \frac{\chi_{\mathrm{L}}}{r_{p}}\left(1-\frac{\chi_{\mathrm{L}}}{\chi_{\mathrm{S}}}\right),
$$

where $\chi_{\mathrm{L}}$ is the comoving distance to the lens, and $\chi_{\mathrm{S}}$ the comoving distance to the source galaxy used to estimate the gravitational shear.

In Fig. 6 we show the forecasted relative error on the SIS free parameter $\sigma_{v}$ for a selection of lens and source sample combinations. We have divided the lens samples into redshift bins of width 0.1 and 0.2 , respectively. It is possible to explore new terrain in galaxy-galaxy lensing already with early SKA1 data by selecting lens samples using spectroscopic redshifts from an HI survey. 


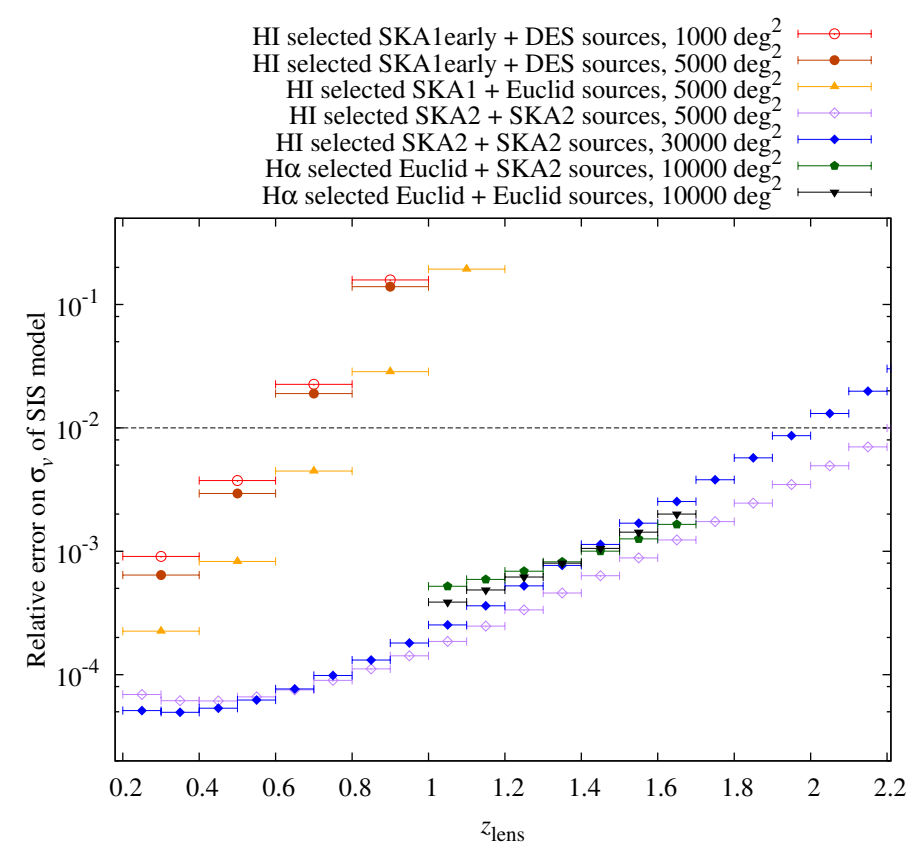

Figure 6: Relative $1 \sigma$ error on the velocity dispersion, $\sigma_{v}$, of a singular isothermal sphere (SIS) profile as a function of the redshift of the lensed objects, constrained via the average tangential shear signal in the transverse separation range $r_{p} \in[0.1 ; 1] \mathrm{Mpc} / h$. The legend lists the lens sample selection, the source sample from which the shear signal is measured, and the assumed overlap in survey area. The error bars show the assumed width of the redshift bins. A $1 \%$ constraint is indicated by the horizontal dashed line.

Combining this with a source sample from an optical survey such as the Dark Energy Survey (DES), one can obtain percent-level constraints out to $z \sim 0.6$ for a few subsamples (e.g. selected on gas abundance). The full SKA1 in combination with Euclid imaging for a source sample will decrease errors further by about a factor of five and extend the range where lenses can be studied to $z=0.8$.

SKA2 will enable unique and powerful galaxy-galaxy lensing measurements without the need to rely on optical counterparts. As Fig. 6 shows, it will generate an unprecedented redshift baseline to study halo properties with a single facility. Out to $z \sim 1.5$ constraints are below the per mil level, which allow for tight constraints on complex models and/or the division into several subsamples. SKA2 will also be on par with Euclid in measuring the galaxy-galaxy lensing signal of emission-line (mostly $\mathrm{H} \alpha$ ) galaxies detected in the Euclid spectroscopic survey, providing independent corroboration for this high-redshift measurement. Note finally that the very deep SKA2 surveys will allow the application of the novel techniques described in Section 2.4 as well as magnification analyses (e.g. Morrison et al. 2012) in a galaxy-galaxy lensing context.

\section{Concluding remarks}

The field of weak lensing with radio surveys is currently in its infancy but the prospect of SKA surveys coming online at the end of this decade presents a huge opportunity for opening a new wavelength window on the dark Universe. We have argued in this Chapter that the radio band 
and the SKA in particular offer exciting prospects for enhancing and extending the reach of weak lensing studies beyond the limits of what is possible using their traditional optical approaches.

The SKA surveys will probe the galaxy population to higher redshift where the lensing signal is larger and potentially easier to measure. There is huge potential to exploit the cross-correlation of lensing shear measurements obtained from overlapping optical and radio surveys which can be powerful in mitigating a number of systematic effects.

Moreover, the radio band offers the exciting possibility of applying new techniques to measure weak lensing signals. One can make use radio polarization and/or rotational velocity information (from HI observations) to mitigate against intrinsic galaxy alignments which are the most worrying systematics for future precision weak lensing measurements. The opportunity to analyze HI Intensity Mapping surveys for the effects of weak lensing will open a new window on the dark Universe at redshifts that are well beyond the reach of traditional cosmic shear techniques.

The SKA also offers a new route to performing galaxy-galaxy lensing where spectroscopic redshifts from $\mathrm{HI}$ observations and the extra diagnostics coming from the radio band will allow for the precise definition of new lens samples.

As with other future large-scale survey instruments, weak lensing studies with the SKA will facilitate the auto- and cross-correlation of galaxy clustering and cosmic shear measurements within the same experimental set up. Generally speaking, the joint exploitation of clustering and lensing is extremely useful as the two probes are highly complementary. Constraints from baryonic acoustic oscillations and/or redshift space distortions are "orthogonal" to weak lensing, especially in the presence of uncertainties in photometric redshifts and inaccurate knowledge of galaxy clustering bias (Zhan 2006; Abate et al. 2012; Laureijs et al. 2011; Amendola et al. 2013).

In terms of constraining power for dark energy and/or modified gravity, galaxy clustering data measure the "dark fluid" equation of state at higher redshift than other standard candles like type Ia supernovae. Furthermore, clustering probes the evolution of matter fluctuations, which means that, through the Poisson equation, it is a proxy for the Newtonian potential $\Phi$. In the case of modified gravity, clustering is sensitive to modifications occurring to the Newtonian gravitational constant. On the other hand, the deflection potential is proportional to the sum of the two metric potentials which are equal in GR but not in more general gravity theories. Through this dependence, lensing effects represent a direct probe of non-standard gravity behaviours. As a consequence, the sensitivity to beyond-GR growth parameters comes in its greatest part from weak lensing, which provides the only direct measurements of growth (without biasing), e.g. Weinberg et al. (2013).

In order to realise the significant and exciting potential that radio weak lensing has to offer, a great deal of development work on analysis techniques, and demonstrating those techniques on real radio data from precursor surveys will be required. A key challenge will be to develop the field of galaxy shape estimation for radio observations to the level of maturity that is currently enjoyed by the optical lensing community. Work has already started in this direction and will gain further momentum through the ongoing "radioGREAT" challenge during the coming year. At the same time, surveys with e-MERLIN, LOFAR and the JVLA are starting to produce the high-resolution, wide-field data suitable for demonstrating radio weak lensing techniques on real observations. The planned program of algorithm development work coupled with the successful application of the developed radio lensing tools to the SKA pathfinder and precursor surveys will position the community favourable to fully exploit the tremendous opportunity for radio weak 
lensing that will be afforded by the commissioning of the SKA at the end of this decade.

\section{References}

Abate A. et al. (LSST Dark Energy Science Collaboration), 2012, arXiv:1211.0310

Amara A., Refregier A., 2008, MNRAS, 391, 228, arXiv:0710.5171

Amendola L. et al., 2013, Living Rev. Relativity 16, 6

Ansari R., Campagne J. E., Colom P., Magneville C., Martin J. M., Moniez M., Rich J., Yeche C., 2012, C. R. Phys., 13, 46

Bartelmann M., Schneider P., 2001, Phys. Reports, 340, 291

Battye R. A., Browne I. W. A., Dickinson C., Heron G., Maffei B., Pourtsidou A., 2013, MNRAS, 434, 1239

Becker R. H., White R. L., Helfand D. J., 1995, ApJ, 450, 559

Bernstein G. M., 2009, ApJ, 695, 652

Blain A. W., 2002, ApJL, 570, L51

Braun R., 2013, "SKA1 Imaging Science Performance", Document number SKA-TEL-SKO-DDXXX, Revision A Draft 2

Brown M. L., Battye R. A., 2011, MNRAS, 410, 2057

Brown M. L., Taylor A. N., Hambly N. C., Dye S., 2002, MNRAS, 333, 501

Brown M. L. et al., 2013, arXiv:1312.5618

Carrillo R. E., McEwen J. D., Wiaux Y., 2014, MNRAS, 439, 3591, arXiv:1307.4370

Catelan P., Kamionkowski M., Blandford R. D., 2001, MNRAS, 320, L7

Chang T.-C., Pen U.-L., Bandura K., Peterson J. B., 2010, Nature, 466, 463

Chang T., Pen U.-L., Bandura K., Peterson J. B., McDonald P., 2008, Phys. Rev. Lett., 100, 091303

Chang T.-C., Refregier A., 2002, ApJ, 570, 447

Chang T., Refregier A., Helfand D. J., 2004, ApJ, 617, 794

Chen X., 2012, Int. J. Mod. Phys., 12, 256

de Jong J. T. A., Verdoes Kleijn G. A., Kuijken K. H., Valentijn E. A., 2013, Exp. Astronomy, 35, 25

Dewdney P., Turner W., Millenaar R., McCool R., Lazio J., Cornewll T., 2013, “SKA1 System

Baseline Design”, Document number SKA-TEL-SKO-DD-001 Revision 1

Gillis, B. et al., 2013, MNRAS, 431, 1439

Heavens A., Refregier A., Heymans C., 2000, MNRAS, 319, 649

Heymans C. et al., 2013, MNRAS, 432, 2433

Hirata C. M., Seljak U., 2004, Phys Rev D, 70, 063526

Hoekstra H., van Waerbeke L., Gladders M. D., Mellier Y., Yee H. K. C., 2002, ApJ, 577, 604

Hu W., 2000, Phys. Rev. D., 62, 3007

Hu W., 2001, ApJ, 557, L79

Huff E. M., Krause E., Eifler T., George M. R., Schlegel D., 2013, arXiv:1311.1489

Joachimi B., Bridle S. L., 2010, A\&A, 523, A1

Kaiser N., 1998, ApJ, 498, 26

Laureijs R. et al., 2011, arXiv:1110.3193 
Lindroos L., Knudsen K. K., Vlemmings W., Conway J., Martí-Vidal I., 2014, MNRAS accepted, arXiv: 1411.1410

Mandelbaum R., Seljak U., Cool R. J., Blanton M., Hirata C. M., Brinkmann J., 2006, MNRAS, 372,758

Mandelbaum R. et al., 2014, ApJS, 212, 5, arXiv:1308.4982

Masui K. W., McDonald P., Pen U.-L., 2010, Phys. Rev. D, 81, 103527

Melchior P., Sutter P. M., Sheldon E. S., Krause E., Wandelt B. D., 2014, MNRAS, 440, 2922

Metcalf R. B., White S. D. M., 2009, MNRAS, 394, 704

Morales M. F., 2006, ApJL, 650, L21

Morrison C. B. et al., 2012, MNRAS, 426, 2489

Morrison G. et al., 2010, ApJS, 188, 178

Muxlow T. W. B. et al., 2005, MNRAS, 358, 1159

Patel P., Bacon D. J., Beswick R. J., Muxlow T. W. B., Hoyle B., 2010, MNRAS, 401, 2572

Patel P., Abdalla F. B., Bacon D. J., Rowe B., Smirnov O., Beswick R. J., 2013, arXiv:1303.4650

Pober J. C. et al., 2013, AJ, 145, 65

Pourtsidou A., Metcalf R. B., 2014, MNRAS, 439, L36

Refregier A., Bacon D. J., 2003, MNRAS 33848

Schinnerer E. et al., 2010, ApJS, 188, 384

Seo H. J., Dodelson S., Marriner J., Mcginnis D., Stebbins A., Stoughton C., Vallinoto A., 2010, ApJ, 721, 164

Stil J. M., Krause M., Beck R., Taylor A. R., 2009, ApJ, 693, 1392

Sutter P. M. et al., 2014, MNRAS, 438, 768, arXiv:1309.1469

Takada M., Jain B., 2004, MNRAS, 348, 897

van Uitert E., Hoekstra H., Franx M., Gilbank D. G., Gladders M. D., Yee H. K. C., 2013, A\&A, 549, A7

Voigt L. M., Bridle, S. L., 2010, MNRAS, 404, 458, arXiv:0905.4801

Voigt L. M., Amara A., Cropper M., Kitching T. D., Massey R., Rhodes J., Schrabback T., 2012, MNRAS, 421, 1385

Weinberg, D. H. et al., 2013, Phys. Rep. 530, 87

Wilman R. J. et al., 2008, MNRAS, 388, 1335

Zhan H., 2006, J. Cosmol. Astropart. Phys., 08, 008

Zahn O., Zaldarriaga, M., 2006, ApJ, 653, 922

Zhang P., Pen, U. L., 2006, MNRAS, 367, 169

Zuntz J. et al., 2014, arXiv:1409.3409

Zwaan M. A. et al., 2003, Astron.J., 125, 2842 